\title{
POLYMER THICK-FILM SENSORS: POSSIBILITIES FOR SMARTCARD BIOMETRICS
}

\author{
Neil J. Henderson*, Thomas V. Papakostas*, Neil M. White* and Pieter H. \\ Hartel** \\ *Department of Electronics and Computer Science, University of Southampton, \\ Highfield, Southampton, SO17 1BJ, UK. **University of Twente, The Netherlands
}

\begin{abstract}
:
In this paper the potential of polymer thick-film sensors are assessed for use as biometric sensors on smartcards. Piezoelectric and piezoresistive sensors have been printed on flexible polyester, then bonded to smartcard blanks. The tactile interaction of a person with these sensors has been investigated. It was found that whilst piezoresistive films offer good dynamic and static sensing properties, the relative complexity of their measurement circuitry over that of piezoelectrics, favours the use of piezoelectric films in the smartcard arena. The outline of a novel biometric discrimination method is presented, for which an equal false-acceptance and falserejection error rate of $2.3 \%$ is reported.
\end{abstract}

\section{Introduction}

In the rapidly growing world of the Internet and e-Commerce, smartcards offer the potential to protect data, sign documents and verify transactions. This illustrates some of the functions of a smartcard driving the motivation to protect and secure access to the smartcard itself. If a smartcard token is to be accepted as the electronic identity of a person, then it is imperative that access to the card's function be restricted to its legitimate user. A method of demonstrating user identity to the smartcard is sought. One promising avenue of research is in the use of biometrics to demonstrate an aspect of the user's identity. Biometrics is a relatively new discipline concerning the use of an individual's physiology or behaviour to verify their identity. It is a growth area attracting a great deal of interest over recent years [1]. Examples of research areas within this field include: the development of biometric sensors to capture fingerprints [2], iris geometries, hand geometry and other physiological quantities. Comparable methods exist for the measurement of behavioural quantities such as voice, typing characteristics and signature recognition [1].

A number of user-verification schemes have been proposed whereby some device external to the smart card captures a biometric quantity, which is subsequently verified on a smartcard platform [3]. Such off-card sensor schemes suffer from the limitation that the external biometric device must be both present and trusted. Furthermore, acceptance of such devices necessitates fundamental and costly modifications to existing worldwide 
infrastructure. An elegant solution to this problem is to embed all components of a user verification scheme on-card.

However, the vast majority of commercial biometric devices have been designed for use in applications where space is not at a premium nor cost a prime concern. Furthermore, it is clear that many existing schemes, such as iris geometry, signature recognition, typing characteristics are not directly applicable to an on-card verification system. The biometric of choice must exploit some accessible aspect of a user's interaction with a card, implying the use of fingerprints, voice, or some form of hand geometry. There are further constraints, however. The desire to embed a verification system imposes severe restrictions on matters such as cost, size, mechanical compliance and processing power. An appropriate sensor must therefore exhibit mechanical flexibility, robustness and miniaturisation in addition to providing a cost-effective solution.

Polymer thick-film (PTF) technology offers many advantages and desirable aspects for use in smartcard biometrics [4]. PTF sensors can be fabricated onto flexible, planar substrates at relatively low temperatures, thereby providing compatibility with the materials used in the production of smartcards. In particular, simple force/pressure sensors can be used to detect the ways in which a user physically interacts with the smartcard. Screen printable piezoelectric and piezoresistive films have been assessed in this respect. A study of the tactile interaction between user and smartcard has been undertaken to identify appropriate verification methods. User interactions are limited but may include a person touching, pressing or tapping the smartcard. We believe this is the first time that polymer thick-films have been used in biometric systems. The results generated show how humans interact with the constraints imposed by embedded smartcard systems.

In this paper we investigate in detail the appropriateness of PTF sensing technology to capture user interactions. We then go on to describe a novel verification system before finally presenting results from user-trials.

\section{The Appropriateness of PTF Sensors}

Figure 1 shows the construction of our piezoelectric sensor, essentially a three-layered device with two silver conductive layers sandwiching an active PZT (85\% Lead Zirconate Titanate and $15 \%$ dielectric) material, measured to be $60 \mu \mathrm{m}$ thick. The piezoresistive sensor (see figure 2) comprises a single resistive layer of RS15114 paste from Electro Science Laboratories, measured to be $20 \mu \mathrm{m}$ thick. During construction, each layer was printed then dried for 10 minutes using an infra-red dryer at $110^{\circ} \mathrm{C}$. Upon completion of the printing process the sensors were cured for 1 hour at $140^{\circ} \mathrm{C}$. The piezoelectric sensor was polarized during the curing process with a poling voltage of $300 \mathrm{~V}$. Wire connections were made using Circuitworks conductive epoxy (CW2400) and 200 $\mu \mathrm{m}$ diameter wire.

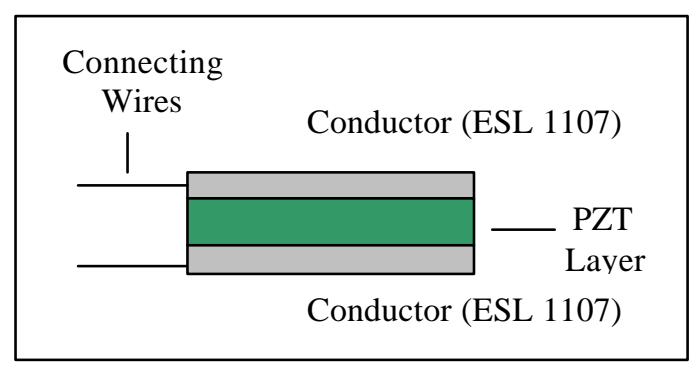

Figure 1: Piezoelectric Sensor Schematic

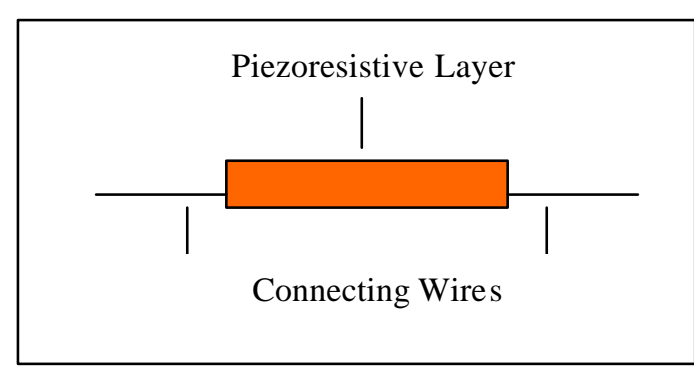

Figure 2: Piezoresistive Sensor Schematic 
Both sensors were printed onto flexible $125 \mu \mathrm{m}$ thick Mylar, which was then cut to approximately equal sensor dimensions - shown in figure 3.

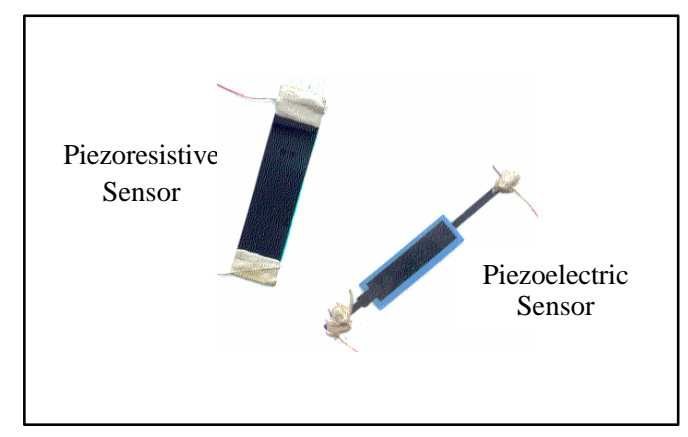

Figure 3: Sensors on Mylar

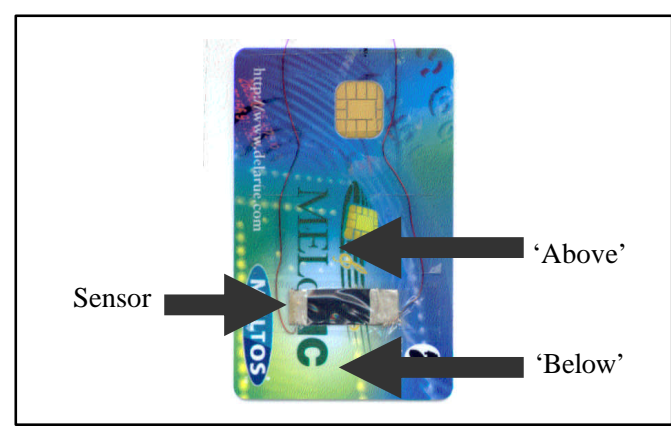

Figure 4: Sensor Position On Smartcard Blank

The sensors were then bonded to smartcard blanks with $50 \mu \mathrm{m}$ Scotch tape - as shown in figure 4.

\subsection{Acquisition Electronics}

In our experiments, piezoresistive measurements were made using a classic resistive (Wheatstone) bridge circuit and amplified using a low-cost Instrumentation Amplifier (AD622 from Analog Devices). Charge generated from the piezoelectric sensor was measured using an ultra-low charge amplifier (LMC6001 from National Semiconductor). Data from both sensors were captured using a Data Translation DT9802 acquisition board, sampling at $5 \mathrm{kHz}$.

\subsection{Capturing Human Interactions}

As indicated in the previous section, the tactile interactions of a person with a smartcard are effectively limited to tapping and pressing. In this section we present the results of an experiment in which we compare the effectiveness of piezoelectric and piezoresistive sensors to capture tapping and pressing interactions.

With the card held in one hand and tapped with the other, figures 5 and 6 show sensor outputs for single taps on the sensor for the piezoelectric and piezoresistive sensors respectively. Both methods capture the dynamics of this interaction adequately, as is clear from curve (1) on each figure. Curves (2) and (3) show the recorded sensor output resulting from taps adjacent to, rather than on, the sensor. Curve (2) results from a tap above the sensor (closer to the centre of the card), whilst curve (3) results from a tap below the sensor (closer to the bottom edge of the card). Although (2) and (3) demonstrate lower outputs than curve (1), they highlight the impracticality of placing two independent sensors on the same card.

Figures 7 and 8 show the piezoelectric and piezoresistive response to a person holding and pressing for a short duration on the card. In common with figures 5 and 6 , curve (1) shows a press on the sensor, whilst curves (2) and (3) show the card being pressed above and below the sensor, respectively. The piezoresistive sensor appears to offer superior response to the slow changing pressure of a finger-press. Interestingly, curve (3) - the piezoresistive response to a finger-press below the sensor is higher than the on-sensor press. Whilst finger-presses are subjective, this further illustrates the problem of placing more than one sensor on the same flexible substrate.

The difference between the quasi-static responses of piezoelectrics and 


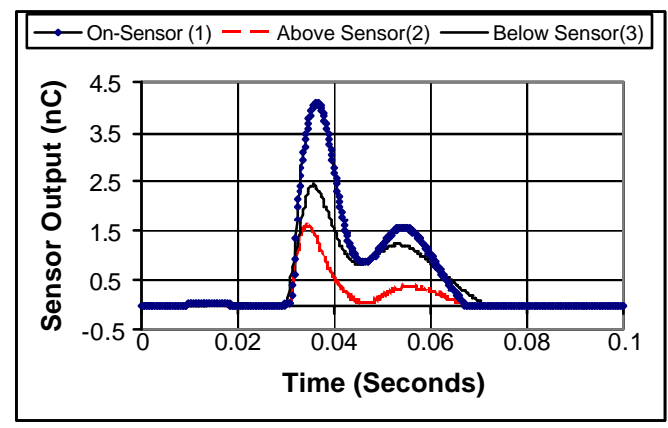

Figure 5: Finger Taps with

Piezoelectric Sensor

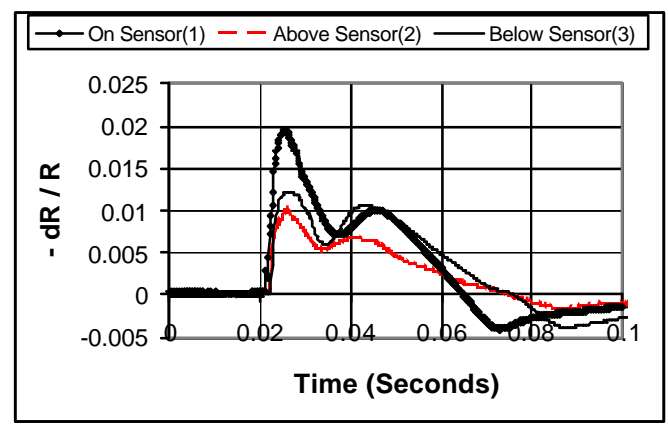

Figure 6: Finger Taps with

Piezoresistive Sensor

piezoresistives is illustrated in figures 9 and 10 which show the response generated by pressing the card for a few seconds. The piezoresistive sensor clearly exhibits better performance in this respect.

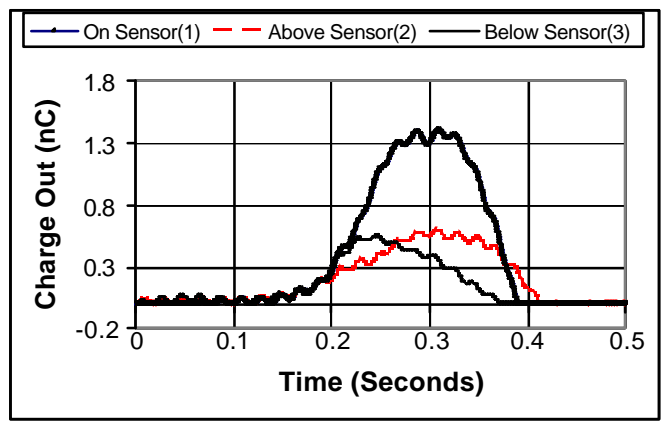

Figure 7: Finger Press with

Piezoelectric Sensor

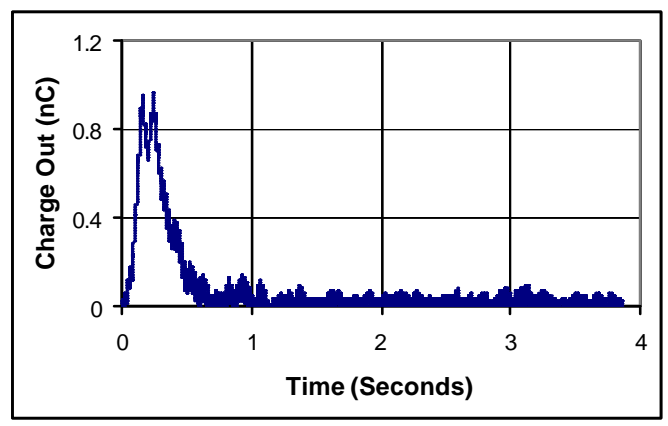

Figure 9: Press and Hold

Piezoelectric Sensor

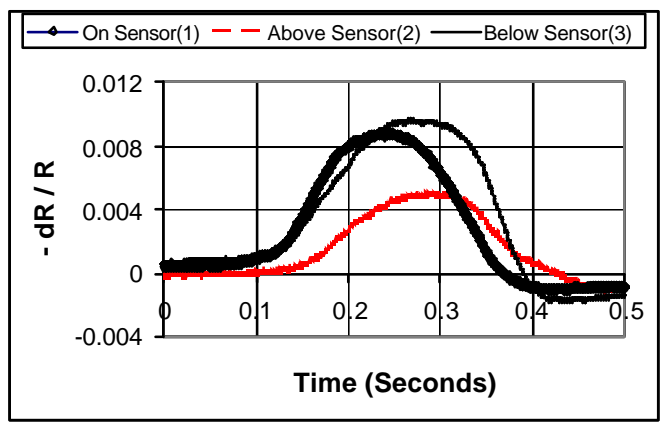

Figure 8: Finger Press with

Piezoresistive Sensor

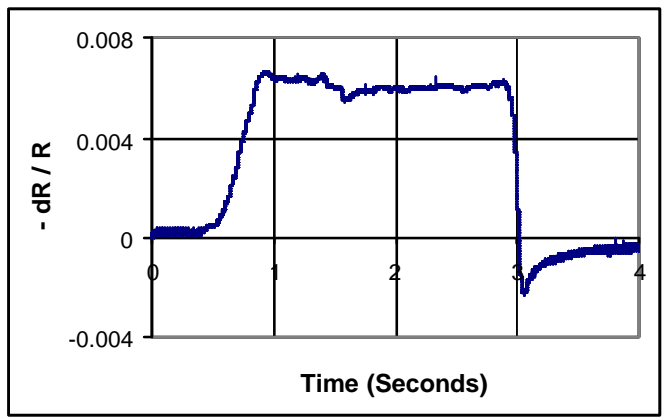

Figure 10: Press and Hold

Piezoresistive Sensor

As a final experiment, the smartcards were placed upon a hard surface, then tapped and pressed. The comparatively small sensor outputs shown in figures 11 and 12 confirm the importance of the flexible substrate on both PTF sensors.

The results presented here indicate that the piezoresistive PTF sensors offer greater versatility than their piezoelectric counterparts. They are able to capture the dynamics of a fast on-off tap, whilst offering good quasi-static measurements. However, their use in the cost-sensitive smartcard arena will be restricted due to the calibration requirements of the sensing bridge arrangements. Balancing the resistances of each arm of the resistive bridge will add time and complexity to the manufacturing process. Whilst piezoelectric PTF sensors offer poor quasi-static measuring properties, they achieve good low-noise dynamic measurement with simple charge amplification. 
The effect of allowing the smartcard substrate to flex on the sensor's output is clearly demonstrated. An unfortunate consequence of this is that multiple pressure sensors on the same flexible substrate are likely to suffer severe cross-sensitivities. It is hence, impractical to use more than one sensor per smartcard.

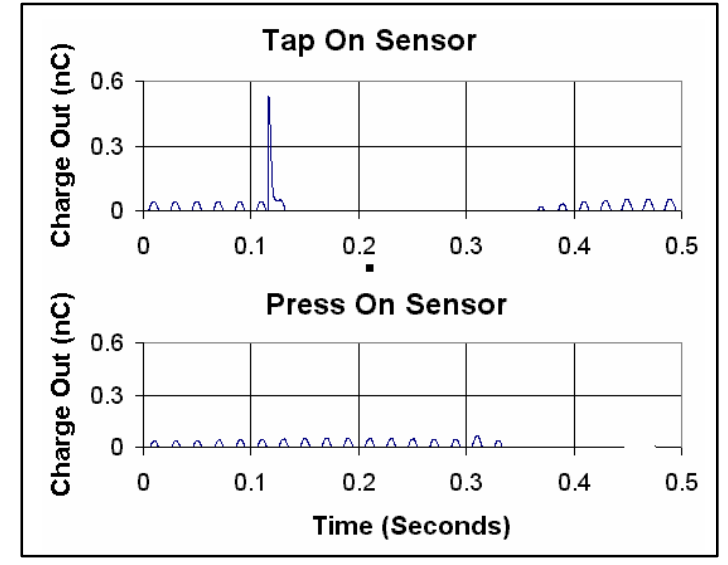

Figure 11: Press and Tap Piezoelectric Sensor - Card On Desk

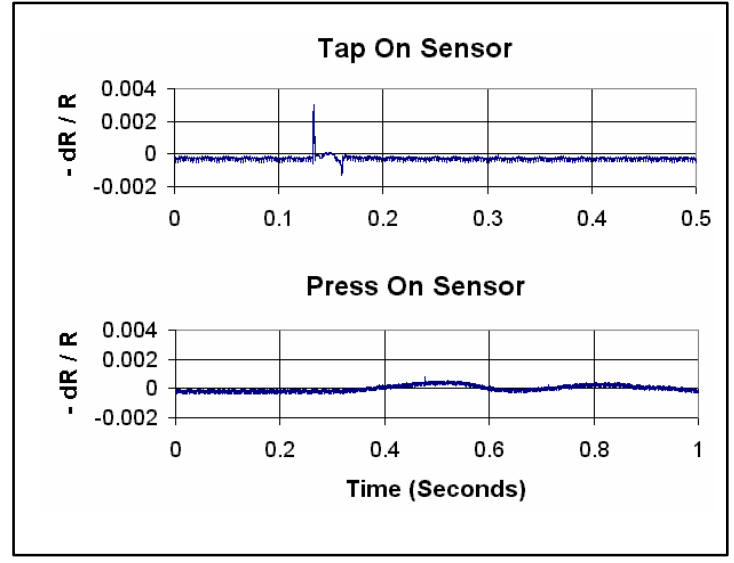

Figure 12: Press and Tap Piezoresistive Sensor - Card On Desk

\section{3. 'Pressure Sequence' - A Novel Smartcard Biometric}

With the above constraints on sensor capabilities and a requirement for simplicity, we look to a very simple technique for the discrimination and hence verification of users. This method exploits the differences with which individuals tap out a rhythm. Much work has been reported on the use of rhythm in keystroke dynamics, whereby identity is verified using an individual's unique pattern of keystrokes [1,5]. We propose a much-reduced form of this mechanism, capturing the pattern of taps on a single sensor, rather than the pattern of keystrokes upon a keyboard. Indeed there is some historical precedent in the use of rhythm, or a series of taps in the recognition of others. For example early telegraphic operators recognised other operators by the way in which they keyed information. Operators developed a distinctive 'fist' or telegraphic style that could be recognised [6].

We dub this method 'Pressure Sequence' and present greater background detail in earlier work [7].

Our validation experiment considered 34 valid users each tapping their own selfselected rhythm on a piezoelectric smartcard pressure sensor (see figure 1). This process provided both the enrolment sequences necessary to generate a reference profile for each user and the verification sequences used in performance testing of our scheme. Eight sequences from each user were employed as the enrolment sequences and a further eight for verification purposes. Eleven impostors provided over 1000 impostor sequences against which the valid sequences were compared.

Using a simple verification technique proposed by Joyce [7] in his work on keystroke dynamics, an equal false acceptance rate (FAR) and false rejection rate (FRR) of $2.3 \%$ was demonstrated. The false rejection rate is defined as the proportion of valid sequences rejected by the system, whereas the false acceptance rate is the proportion of impostor sequences, falsely accepted. Plotting FAR against FRR, one can assess the overall system performance. This is given in Figure 13 and known as the receiver operating characteristics (ROC), for the system. Detailed discussion of the verification methods used and their processing requirements will appear in future work. 


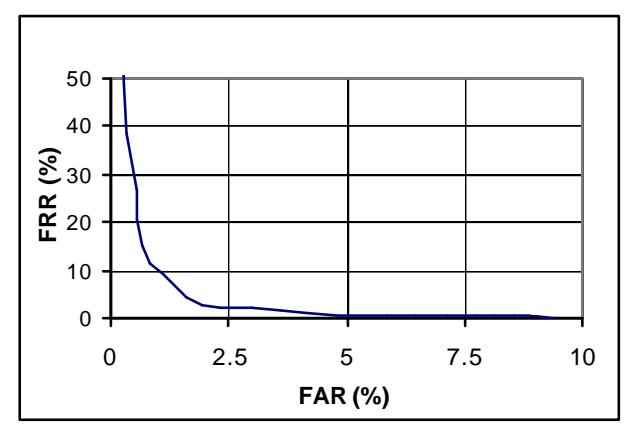

Figure 13: Pressure Sequence ROC Curve

\section{Conclusions}

In this paper we have identified polymer thick-film sensors as a low-cost, robust and mechanically compliant technology for embedded biometric sensors on smartcards. We reasoned that a biometric system on a smartcard should capitalise on some natural aspect of a person's tactile interaction with the card and found this to be essentially limited to finger pressing and tapping. Two potential sensing methods; namely piezoelectric and piezoresistive were assessed in this respect.

It was found that whilst both sensing methodologies proved useful in measuring the dynamics of a fast-changing signal such as a tap, slow quasi-static signals are best measured with piezoresistive films. Furthermore, we reported on the contribution of substrate flex as an important agent in the sensitivities of both sensing regimes. An undesirable consequence of this is that multiple sensors on the same substrate are likely to suffer severe crosssensitivities. As a result practical systems will be limited to one single sensor.

Whilst piezoresistive films offer improved sensing scope over piezoelectric films, we favour the use of piezoelectrics due to the simplicity of their sensing circuitry.

We finally discussed the implementation of a novel verification scheme, whereby identity is verified by means of a sequence of taps on a piezoelectric sensor. We offer justification for this method and present its discrimination potential. From a population of 34 valid users and over 1000 impostor sequences, we found an equal-error rate of just $2.3 \%$, comparing favourably with a number of other biometric schemes.

\section{References:}

[1] Jain A, Bolle R, Pankanti S (Editors) "Biometrics: Personal Identification in Networked Society", Klewer 1998, Boston. ISBN 0792383451

[2] Tartagni M, Guerrieril R, "A Fingerprint Sensor Based on the Feedback Capacitive Sensing Scheme" IEEE Journal of Solid State Circuits V33, N1, January 1998

[3] Wirtz, B. "Biometric Systems", Secure, The Silicon Trust Quarterly Report, Autumn 2000

[4] Papakostas T, White NM, "Polymer Thick Film Sensors and Applications" Sensors and their Applications X Cardiff, UK. pp 125-130

[5] Joyce, Gupta "Identity Authentication Based on Keystroke Dynamics" Communications of the ACM V33, N2, pp168-176, 1990

[6] Bryan WL, Harter N, "Studies in the Physiology and Psychology of the Telegraphic Language" Psychological Review, 1897, Vol. 4, pp 27-53

[7] Henderson, N. Hartel, P. "'Pressure Sequence' - A Novel Method of Protecting Smart Cards." Smart Card Research and Advanced Applications. IFIP/TC8 September 20-22, 2000, Bristol, UK. Kluwer Academic Publishers, Boston. ISBN 0-7923-7953-5 